\title{
A phase transition in energy-filtered RNA secondary structures
}

\author{
Hillary S. W. Han and Christian M. Reidys* \\ Department of Mathematics and Computer Science \\ University of Southern Denmark, Denmark \\ Phone: *45-24409251 \\ Fax: *45-65502325 \\ email:duck@santafe.edu
}

\begin{abstract}
In this paper we study the effect of energy parameters on minimum free energy (mfe) RNA secondary structures. Employing a simplified combinatorial energy model, that is only dependent on the diagram representation and that is not sequence specific, we prove the following dichotomy result. Mfe structures derived via the Turner energy parameters contain only finitely many complex irreducible substructures and just minor parameter changes produce a class of mfe-structures that contain a large number of small irreducibles. We localize the exact point where the distribution of irreducibles experiences this phase transition from a discrete limit to a central limit distribution and subsequently put our result into the context of quantifying the effect of sparsification of the folding of these respective mfe-structures. We show that the sparsification of realistic mfe-structures leads to a constant time and
\end{abstract}


space reduction and that the sparsifcation of the folding of structures with modified parameters leads to a linear time and space reduction. We furthermore identify the limit distribution at the phase transition as a Rayleigh distribution.

Keywords: RNA secondary structure, loops-based, energy model, dominant singularity, limit distribution

\section{InTRODUCTION}

An RNA molecule is described by its primary structure, a linear string composed of the nucleotides $\mathbf{A}, \mathbf{G}, \mathbf{U}$ and $\mathbf{C}$, referred to as the backbone. Each nucleotide can form a base pair by interacting with at most one other nucleotide by establishing hydrogen bonds. Here we restrict ourselves to Watson-Crick base pairs GC and AU as well as the wobble base pairs GU. In the following, base triples as well as other types of more complex interactions are neglected. RNA structures can be presented as diagrams by drawing the backbone horizontally and all base pairs as arcs in the upper halfplane; see Figure 1. This set of arcs is tantamount to our notion of coarsegrained RNA structure. In particular, we shall ignore any spatial embedding or geometry of the molecule beyond its collection of base pairs. Accordingly, particular classes of base pairs translate into specific structure categories, the most prominent of which are secondary structures [9, 12, 16, 17]. When represented as diagrams, secondary structures have only non-crossing base pairs (arcs). In the following an 


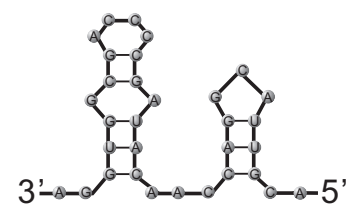

(a)

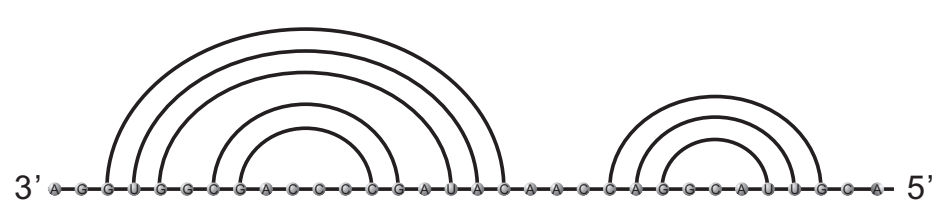

(b)

FiguRE 1. (A) An RNA secondary structure and (B) its diagram representation.

RNA secondary structure is tantamount to a diagram without any crossing arcs. The combinatorics and prediction of RNA secondary from primary structure was pioneered three decades ago by Michael Waterman [17, 16, 17, 18].

RNA structures are a result of a folding of the primary sequence. The folded configurations are energetically somewhat optimal. Here energy means free energy, which is dominated by the loops forming between adjacent base pairs and not by the hydrogen bonds of the individual base pairs [10]. In addition sterical constraints imply certain minimum arc-length conditions for minimum free energy configurations [14].

For a given RNA sequence polynomial-time dynamic programming (DP) algorithms can be devised, finding such minimal energy configurations. The most commonly used tools predicting simple RNA secondary structure mfold [20] and the Vienna RNA Package [6], are running at $O\left(N^{2}\right)$ space and $O\left(N^{3}\right)$ time solution.

In the context of polynomial-time DP algorithms a particular method, the sparsification has been devised. Sparsification is tailored to speed up DP-algorithms 
predicting minimum free energy (mfe)-secondary structures [19, 1] by pruning certain computation paths encountered in the DP-recursions, see Fig. 2. In the context

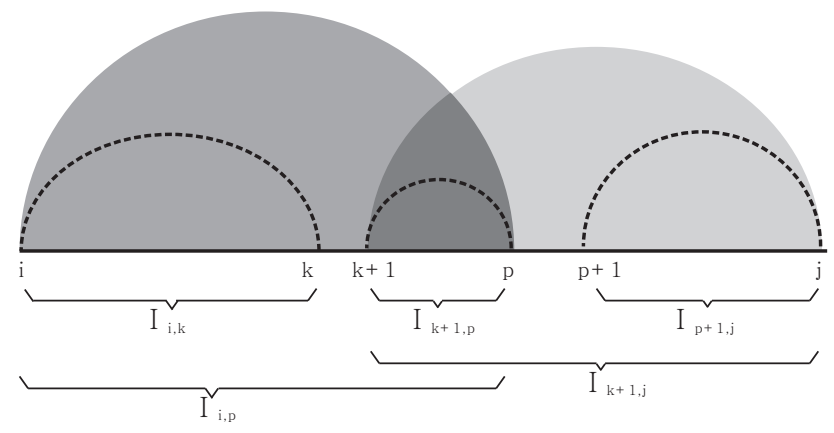

FiguRE 2. Sparsification: suppose the optimal solution $I_{i, j}$ is obtained from the optimal solutions $I_{i, k}, I_{k+1, p}$ and $I_{p+1, j}$. Based on the recursions of the secondary structures, $I_{i, k}$ and $I_{k+1, p}$ produce an optimal solution of $I_{i, p}$. Similarly, $I_{k+1, p}$ and $I_{p+1, j}$ produce an optimal solution of $I_{k+1, j}$. Now, in order to obtain an optimal solution of $I_{i, j}$ it is sufficient to consider either the grouping $I_{i, p}$ and $I_{p+1, j}$ or $I_{i, k}$ and $I_{k+1, j}$.

of the folding of RNA secondary structures, sparsification reduces the DP-recursion paths to be based on so called candidates. A candidate is in this case an interval, for which the optimal solution cannot be written as a sum of optimal solutions of subintervals. Tracing back these candidates gives rise to irreducible structures and the crucial observation is here that these irreducibles appear only at a low frequency. This means that there are only relatively few candidates have to be considered, which in turn implies a significant reduction in time and space complexity.

In this paper we study the effect of the particular choice of energy parameters on sparsification. In other words, we study what happens to RNA structures of a 
certain energy and quantify the effect of sparsification of their mfe-folding. We shall see that this energy filtration of secondary structures can have a dramatic effect on the structures having significant implication for the effect of sparsification.

The energy parameters are associated to the loops of a secondary structure, they are empirically measured enthalpic and entropic terms that depend on loop sequence, length and type [13, 10]. We shall restrict ourselves to a simplified notion of energy that does not take into account the specifics of nucleotides but only depends on the combinatorial representation the secondary structure. For instance, the free energy of a hairpin loop $H, G_{H}$, is given by

$$
G_{H}=\alpha_{1}+\alpha_{2} \ell_{H}+\alpha_{3} \text { and } Q_{H}=v^{G_{H}},
$$

$\alpha_{1}$ being the penalty for forming $H, \alpha_{2}$ the penalty for an unpaired base, $\alpha_{3}$ the score associated to a tetra-loop, $\ell_{H}$ denoting the number of unpaired bases and $Q_{H}$ being the weight of $H$. The other two loop-types are treated along these lines.

Equipped with this notion of "combinatorial" energy we study the energy filtration of RNA secondary structures. In light of the above discussion about the candidates and sparsification we pay particular attention to irreducible RNA secondary structures. One key question here being: under which conditions does the probability of finding an irreducible minimum-free energy structure tend to zero for larger and larger sequences? 
The main results of this paper are as follows: we will show that

- for energy-parameters mimicking the established Turner energy model [10] sparsification implies a time and space reduction by a constant factor,

- there exist energy-parameters close to those of the Turner energy model [10] for which sparsification implies a linear time reduction,

- the effect of sparsification is closely connected to the distribution of irreducibles within secondary structures. To be precise we prove that this distribution undergoes a phase transition from a discrete limit law to a central limit law,

- the limit distribution of irreducibles at the phase transition is a Rayleigh-law and a DP-folding in this regimen experiences a linear reduction in space and time.

\section{Some BASIC FACTS}

As mentioned above, we present an RNA secondary structure as a diagram by drawing its backbone as a horizontal line containing vertices corresponding to the labels of the nucleotides and each Watson-Crick base pair as an arc or chord in the upper halfplane. Consequently, the diagram representation ignores the particular type of nucleotides. Any such pair may be inserted in two positions $i, j$ incident to an arc as long as it is compatible with the Watson-Crick and $\mathbf{G}-\mathbf{U}$ base paring rules. 
The length of one chord $(i, j)$ is $\ell=j-i$ and in this paper, we only consider RNA secondary structure with chord length $\geq 4$, see Fig. 3. A chord connecting the first and last vertices is called a rainbow and a RNA secondary structures exhibiting a rainbow is an irreducible RNA secondary structure. This notion of irreducibility comes up naturally when one decomposes a structure by means of cutting the backbone in two positions without breaking any arcs. Let $\mathscr{S}(n)$ and $\mathscr{C}(n)$ denote the collections

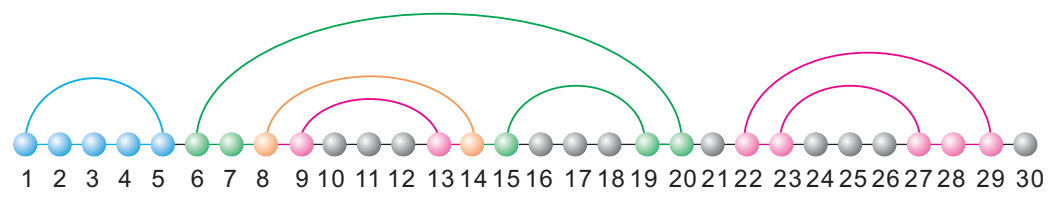

FiguRE 3. A diagram representation of secondary structure with $\geq 4$.

of all linear chord diagrams of RNA secondary structures and irreducible secondary structures on $n$ vertices, respectively. Let $\mathbf{s}(n)$ and $\mathbf{c}(n)$ denote the cardinalities of these sets. Furthermore, setting $\mathcal{S}=\cup_{n \geq 0} \mathscr{S}(n)$ and $\mathcal{C}=\cup_{n \geq 0} \mathscr{C}(n)$ denote the set of all linear chord diagrams of RNA secondary structures and irreducible secondary structures, let $s \in \mathcal{S}$ and $c \in \mathcal{C}$ denote a $\mathcal{S}$ - or $\mathcal{C}$-structure.

Let $\mathscr{S}(n, j, i) \supseteq \mathscr{C}(n, j, i)$ denote the collections of RNA secondary structures and irreducible RNA secondary structures on $n \geq 0$ vertices having length $n$, energy $j$ and weight $i$, then

$$
\begin{aligned}
\mathbf{S}(z, v, p) & =\sum_{n \geq 0} \sum_{j} \sum_{i \geq 0} \mathbf{s}(n, j, i) z^{n} v^{j} p^{i} \\
\mathbf{C}(z, v, p) & =\sum_{n \geq 0} \sum_{j} \sum_{i \geq 0} \mathbf{c}(n, j, i) z^{n} v^{j} p^{i} .
\end{aligned}
$$


Thermodynamic models for nucleic acid secondary structure are based on a decomposition of the base-pairing diagram of structures into distinct loops that are associated with empirically measured enthalpic and entropic terms that depend on loop sequence, length and type [13, 10]. To obtain a better idea about these loops we give the diagram representations of three types of Loops $L$ :

- a Hairpin-loop $(H)$ consists of a chord $(i, j)$ with a sequence of unpaired bases $[i+1, j-1]$. In particular, we have the restriction $j-i \geq 4$; if $j-i=4$ (tetra-loop);

- an interior-loop $(I)$ consists of two base pairs $(i, j)$ and $\left(i_{1}, j_{1}\right)$ and three sequence of unpaired bases $\left[i+1, i_{1}-1\right],\left[j_{1}+1, j-1\right]$ and $\left[i_{1}-1, j_{1}-1\right]$. The case of $i+1=i_{1}-1$ and $i_{1}-1=j_{1}-1$ is referred to as helix;

- a multi-loop $(M)$ is a sequence:

$$
\left((i, j),\left(i_{1}, j_{1}\right), \ldots,\left(i_{k}, j_{k}\right),\left[i, i_{1}\right],\left[j_{k-1}+1, i_{k}-1\right],\left[j_{k}+1, j\right]\right)
$$

with sequences of unpaired bases $\left[j_{k-1}+1, i_{k}-1\right]$, for $k \geq 2$, see Fig. 4 .

The free energy $G_{s}$ of a secondary structure $s$ is the sum of the energies of its constituent Loops $L$ [10]. Thus the total free energy $G_{s}$ is given by $G_{s}=\sum_{L \in s} G_{L}$. This notion of energy allows one to compute, by means of dynamic programming [6. 21] the minimum free energy configurations as well as the partition function [1] as a weighted sum $Q=\sum_{s \in \mathcal{S}} e^{G_{s} / R T}$, where $\mathrm{R}$ is the universal gas constant, $T$ is the 
hairpin-loop
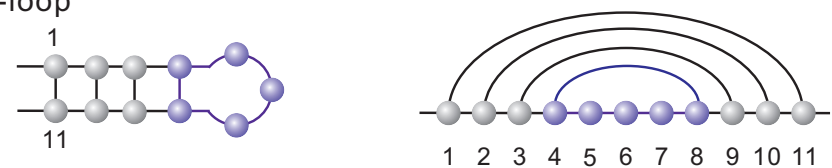

interior-loop
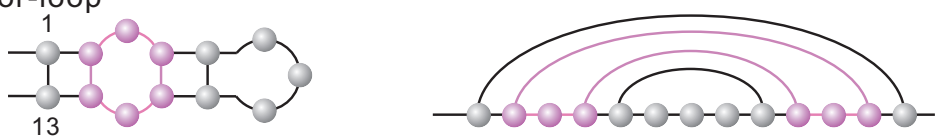

$\begin{array}{lllllllllllllllll}1 & 2 & 3 & 4 & 5 & 6 & 7 & 8 & 9 & 10 & 11 & 12 & 13\end{array}$

multi-loop
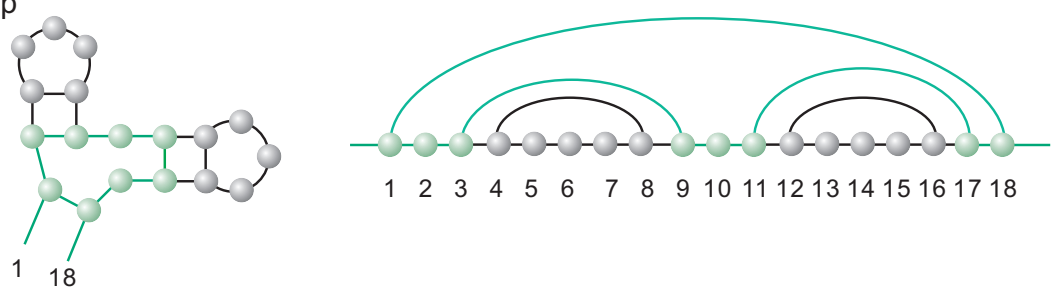

FiguRE 4. It shows hairpin-loop $H$, interior-loop $I$ and multi-loop $M$.

temperature and $e^{G_{s} / R T}$ is the weight of sampling a secondary structure $s$ with free energy $G_{s}$.

In the following we consider a notion of energy that does not take into account the specifics of nucleotides and only depends on the combinatorial representation the secondary structure. Our energy model is based on seven parameters $\mathcal{P}=$ $\left\{\alpha_{1}, \alpha_{2}, \alpha_{3}, \beta_{1}, \beta_{2}, \gamma_{1}, \gamma_{2}\right\}$ defined as follows:

- hairpin-loop $H$ :

$$
G_{H} \sim \alpha_{1}+\alpha_{2} \ell_{H}+\alpha_{3} \text { and } Q_{H}=v^{G_{H}},
$$


where $G_{H}$ is the free energy of $H, \alpha_{1}$ is the penalty for forming $H, \alpha_{2}$ is the penalty for an unpaired base and $\alpha_{3}$ is a score associated to a tetra-loop. Furthermore $\ell_{H}$ denotes the number of unpaired bases and $Q_{H}$ is the weight of $H$.

\section{- Interior-loop $I$ :}

$$
G_{I} \sim \beta_{1}+\ell_{I} \beta_{2} \quad \text { and } \quad Q_{I}=v^{G_{I}}
$$

where $G_{I}$ is free energy of $I, \beta_{1}$ is a favorable bonus of a helix and $\beta_{2}$ is the penalty of an unpaired base of $I$. Furthermore $\ell_{I}$ denotes the total number of unpaired bases of $I$ and $Q_{I}$ is the weight of $I$.

- Multi-loop $M$ :

$$
G_{M} \sim \gamma_{1}+B \gamma_{2}+U \times 0 \quad \text { and } \quad Q_{M}=v^{G_{M}}
$$

$G_{M}$ is the free energy of $M, \gamma_{1}$ is the penalty for the formation of a multiloop, $B$ is the number of base pairs defining the multiloop (including the closing pair $i \cdot j$ ), and $U$ is the number of unpaired bases in the multiloop; $\gamma_{2}$ is the penalty for the base pair defining the multiloop and $Q_{M}$ is the weight of one $M$.

This energy model induces the partition function

$$
Q=\sum_{s \in \mathscr{S}(n)} v^{G_{s}}
$$


where $v^{G_{s}}$ the weight for sampling a secondary structure with free energy $G_{s}$.

\section{ENERGY FILTRATION}

Theorem 1. The trivariate generating function $\mathbf{C}(z, v, p)$ counting $R N A$ secondary structures having length $n$, energy $i$ and $j$ arcs, satisfies the following recursion:

$$
\begin{aligned}
\mathbf{C}(z, v, p) & =p v^{\alpha_{1}} z^{2}\left(\frac{\left(z v^{\alpha_{2}}\right)^{3}}{1-z v^{\alpha_{2}}}+z^{4} v^{\alpha_{3}}-z^{4} v^{4 \alpha_{2}}\right) \\
& +p \frac{z^{2} v^{\beta_{1}}}{\left(1-z v^{\beta_{2}}\right)^{2}} \mathbf{C}(z, v, p)+p \frac{v^{\gamma_{1}}\left(z^{2} v^{\gamma_{2}}\right)\left(\mathbf{C}(z, v, p) v^{\gamma_{2}}\right)^{2}}{\left((1-z)^{3}-\mathbf{C}(z, v, p) v^{\gamma_{2}}(1-z)^{2}\right)}
\end{aligned}
$$

The proof is a straightforward exercise in symbolic methods and will consequently be omitted.

We shall pass from the trivariate generating function to univariate ones by specifying the indeterminants $v$ and $p$ as follows: since there are a total of four nucleotides $\mathbf{A}$, $\mathbf{G}, \mathbf{U}$ and $\mathbf{C}$ and only six of base pairs, namely $\mathbf{G C}, \mathbf{C G}, \mathbf{A} \mathbf{U}, \mathbf{U A}, \mathbf{U G}$ and $\mathbf{G U}$, we set $p=\frac{6}{16}$. This choice reflects the probability of randomly forming a (valid) base pair. We proceed similarly for the indeterminant $v$ setting $v=e^{\frac{1}{R T}} \approx 1.843868184$.

These two choices induce the univariate generating functions $\mathbf{C}^{*}(z)=\sum_{n \geq 0} \mathbf{c}^{*}(n) z^{n}$, $\mathbf{S}^{*}(z)=\sum_{n \geq 0} \mathbf{s}^{*}(n) z^{n}$ and the sets of $\mathcal{S}^{*}$ and $\mathcal{C}^{*}$, which are weighted set of $\mathcal{S}$ and $\mathcal{C}$, separately. Furthermore, $\mathbf{c}^{*}(n)$ or $\mathbf{s}^{*}(n)$ denote the summation of energy weight of 
all the $\mathcal{C}^{*}$-structures or $\mathcal{S}^{*}$-structures with length $n$.

We have $\mathbf{c}^{*}(n)=0, \mathbf{s}^{*}(n)=0$, for $n=0, \ldots, 4$; and $\mathbf{c}^{*}(n)>0, \mathbf{s}^{*}(n)>0$, for $n \geq 5$, see Claim 0 of Lemma 2.

Symbolic methods immediately imply

Theorem 2. The bivariate generating function $\mathbf{S}^{*}(z)$ is given by:

$$
\mathbf{S}^{*}(z)=\frac{1}{1-\left(z+\mathbf{C}^{*}(z)\right)}
$$

and furthermore, $\mathbf{S}^{*}(z, t)$ is

$$
\mathbf{S}^{*}(z, t)=\frac{1}{1-\left(z+t \mathbf{C}^{*}(z)\right)}
$$

The key for the following analysis is to study the dominant singularities of $\mathbf{C}^{*}(z)$ and $\mathbf{S}^{*}(z)$. It is their relative location and behavior that is responsible for the observed limit distributions as well as the effect of sparsification.

We begin our analysis by making the following observations. Theorem 1 implies

$$
w_{2}^{*}(z) \mathbf{C}^{*}(z)^{2}+w_{1}^{*}(z) \mathbf{C}^{*}(z)+w_{0}^{*}(z)=0
$$


where

$$
\begin{aligned}
& w_{2}^{*}(z)=\left(16 v^{\gamma_{2}}(1-z)^{2}+6 z^{2} v^{\gamma_{1}+3 \gamma_{2}}\right)\left(1-z v^{\alpha_{2}}\right)\left(1-z v^{\beta_{2}}\right)^{2} \\
&-6 z^{2} v^{\beta_{1}+\gamma_{2}}(1-z)^{2}\left(1-z v^{\alpha_{2}}\right) \\
& w_{1}^{*}(z)=6 z^{2} v^{\beta_{1}}(1-z)^{3}\left(1-z v^{\alpha_{2}}\right)-6 v^{\alpha_{1}+\gamma_{2}+3 \alpha_{2}} z^{5}\left(1-z v^{\beta_{2}}\right)^{2}(1-z)^{2}+6 z^{6} v^{\alpha_{1}+\gamma_{2}} \\
& \quad\left(v^{4 \alpha_{2}}-v^{\alpha_{3}}\right)(1-z)^{2}\left(1-z v^{\alpha_{2}}\right)\left(1-z v^{\beta_{2}}\right)^{2}-16(1-z)^{3}\left(1-z v^{\alpha_{2}}\right)\left(1-z v^{\beta_{2}}\right)^{2} \\
& w_{0}^{*}(z)=6 v^{\alpha_{1}} z^{5}(1-z)^{3}\left(1-z v^{\beta_{2}}\right)^{2}\left(v^{3 \alpha_{2}}-z\left(v^{4 \alpha_{2}}-v^{\alpha_{3}}\right)\left(1-z v^{\alpha_{2}}\right)\right) .
\end{aligned}
$$

We observe that only one solution of eq. (3.4) has the property $\mathbf{c}^{*}(n)>0$, namely

$$
\mathbf{C}^{*}(z)=\frac{N^{*}(z)}{D^{*}(z)}
$$

where $N^{*}(z)=-w_{1}^{*}(z)+\sqrt{w_{1}^{*}(z)^{2}-4 w_{2}^{*}(z) w_{0}^{*}(z)}$ and $D^{*}(z)=2 w_{2}^{*}(z)$.

Let $\rho_{c^{*}}$ denote the radius of convergence of $\mathbf{C}^{*}(z)$ and $\rho_{s^{*}}$ denote the radius of convergence of $\mathbf{S}^{*}(z)$. Then, clearly, $0<\rho_{c^{*}}<1$ and in view of $\mathcal{C}^{*} \subset \mathcal{S}^{*}$, we have $0<\rho_{s^{*}} \leq \rho_{c^{*}}<1$.

- Let furthermore $\rho_{r}$ denote the minimum positive real root of odd order of the discriminant

$$
w_{1}^{*}(z)^{2}-4 w_{2}^{*}(z) w_{0}^{*}(z)=0
$$

- Let $\rho_{d}$ and $\rho_{p}$ denote the minimum positive real roots of the equations

$$
w_{2}^{*}(z)=0 \quad \text { and } \quad 1-\left(z+\mathbf{C}^{*}(z)\right)=0 .
$$


Theorem 3. (Pringsheim's theorem)[15]: If $f(z)$ is representable at the origin by a series expansion that has non-negative coefficients and radius of convergence $r$, then the point $z=r$ is a dominant singularity of $f(z)$.

Lemma 1. For the positive dominant real singularities of $\mathbf{C}^{*}(z)$ and $\mathbf{S}^{*}(z), \rho_{c^{*}}$ and $\rho_{s^{*}}$, we distinguish the following three cases:

(I): $\rho_{c^{*}}=\rho_{r}$ and $\rho_{s^{*}}=\rho_{c^{*}}$;

(II): $\rho_{c^{*}}=\rho_{r}$ and $\rho_{s^{*}}=\rho_{p}$, where $\rho_{s^{*}}<\rho_{c^{*}}$;

(III): $\rho_{c^{*}}=\rho_{r}$ and $\rho_{s^{*}}=\rho_{p}$, where $\rho_{s^{*}}=\rho_{c^{*}}$.

Proof. According to Theorem 3, we have $\rho_{c^{*}}=\min \left\{\rho_{r}, \rho_{d}\right\}$.

Claim 0: We have $\rho_{d} \neq \rho_{r}$.

To prove Claim 0 . We begin by observing that $v^{4 \alpha_{2}}-v^{\alpha_{3}}<0$, since $\alpha_{2}<0, \alpha_{3}>0$ and $v>1$, then for arbitrary real $z, 0<z<1$,

$$
w_{0}^{*}(z)=6 v^{\alpha_{1}} z^{5} \underbrace{(1-z)^{3}\left(1-z v^{\beta_{2}}\right)^{2}}_{>0}(v^{3 \alpha_{2}}-z \underbrace{\left(v^{4 \alpha_{2}}-v^{\alpha_{3}}\right)}_{<0} \underbrace{\left(1-z v^{\alpha_{2}}\right)}_{>0})>0 .
$$

Suppose $\rho_{d}=\rho_{r}$. Then, in view of eq. (3.5)

$$
w_{2}^{*}\left(\rho_{d}\right)=0 \quad \text { and } \quad w_{1}^{*}\left(\rho_{d}\right)^{2}-4 w_{2}^{*}\left(\rho_{d}\right) w_{0}^{*}\left(\rho_{d}\right)=0
$$

and consequently $w_{1}^{*}\left(\rho_{d}\right)=0$. But in this case eq. (3.4) implies $w_{0}^{*}\left(\rho_{d}\right)=0$, which conflict with $w_{0}^{*}\left(\rho_{d}\right)>0$ and Claim 0 is proved. 
First, we discuss the case $\rho_{d}<\rho_{r}$.

Claim 1: $\rho_{d}$ is a removable singularity of $\mathbf{C}^{*}(z)$.

To prove Claim 1. Suppose first $N^{*}\left(\rho_{d}\right) \neq 0$, since $D^{*}\left(\rho_{d}\right)=0$, thus $\lim _{z \rightarrow \rho_{d}} \mathbf{C}^{*}(z)=$ $\infty$, so $\rho_{d}$ is accordingly a pole of $\mathbf{C}^{*}(z)$.

In view of

$$
\mathbf{S}^{*}(z)^{-1}=1-\left(z+\frac{N^{*}\left(\rho_{d}\right)}{D^{*}\left(\rho_{d}\right)}\right)
$$

we have $\lim _{z \rightarrow \rho_{d}} \mathbf{S}^{*}(z)=0$, this is impossible, since $\mathbf{S}^{*}(z)=\sum_{n \geq 0} \mathbf{s}^{*}(n) z^{n}$, the only value of $\rho_{d}$ to make $\lim _{z \rightarrow \rho_{d}} \mathbf{S}^{*}(z)=0$ is $\rho_{d}=0$.

Thus $N^{*}\left(\rho_{d}\right)=0$ holds, we compute using $D^{*}\left(\rho_{d}\right)=2 w_{2}^{*}\left(\rho_{d}\right)=0$,

$$
\begin{aligned}
N^{*}\left(\rho_{d}\right) & =-w_{1}^{*}\left(\rho_{d}\right)+\sqrt{w_{1}^{*}\left(\rho_{d}\right)^{2}-4 w_{2}^{*}\left(\rho_{d}\right) w_{0}^{*}\left(\rho_{d}\right)} \\
& =-w_{1}^{*}\left(\rho_{d}\right)+\operatorname{csgn}\left(w_{1}^{*}\left(\rho_{d}\right)\right) \cdot\left(w_{1}^{*}\left(\rho_{d}\right)\right)=0 .
\end{aligned}
$$

Consequently, $\operatorname{csgn}\left(w_{1}^{*}\left(\rho_{d}\right)\right)$ is +1 and we have

$$
\begin{aligned}
& \lim _{z \rightarrow \rho_{d}} \mathbf{C}^{*}(z) \\
= & \lim _{z \rightarrow \rho_{d}} \frac{\left(-w_{1}^{*}(z)+\sqrt{w_{1}^{*}(z)^{2}-4 w_{2}^{*}(z) w_{0}^{*}(z)}\right)\left(-w_{1}^{*}(z)-\sqrt{w_{1}^{*}(z)^{2}-4 w_{2}^{*}(z) w_{0}^{*}(z)}\right)}{2 w_{2}^{*}(z)\left(-w_{1}^{*}(z)-\sqrt{w_{1}^{*}(z)^{2}-4 w_{2}^{*}(z) w_{0}^{*}(z)}\right)} \\
= & \lim _{z \rightarrow \rho_{d}} \frac{2 w_{0}^{*}(z)}{\left(-w_{1}^{*}(z)-\sqrt{w_{1}^{*}(z)^{2}-4 w_{2}^{*}(z) w_{0}^{*}(z)}\right)} \\
= & \frac{2 w_{0}^{*}\left(\rho_{d}\right)}{\left(-w_{1}^{*}\left(\rho_{d}\right)-w_{1}^{*}\left(\rho_{d}\right)\right)}=-\frac{w_{0}^{*}\left(\rho_{d}\right)}{w_{1}^{*}\left(\rho_{d}\right)} .
\end{aligned}
$$


Since $w_{2}^{*}\left(\rho_{d}\right)=0$ and $\rho_{d} \neq \rho_{r}$, hence $w_{1}^{*}\left(\rho_{d}\right)^{2}-4 w_{0}^{*}\left(\rho_{d}\right) w_{2}^{*}\left(\rho_{d}\right) \neq 0$, whence $w_{1}^{*}\left(\rho_{d}\right) \neq$ 0 . Using our previous observation $w_{0}^{*}\left(\rho_{d}\right)>0$, we conclude

$$
\lim _{z \rightarrow \rho_{d}} \mathbf{C}^{*}(z)=-\frac{w_{0}^{*}\left(\rho_{d}\right)}{w_{1}^{*}\left(\rho_{d}\right)} \neq 0, \infty
$$

which implies that $\rho_{d}$ is a removable singularity, whence Claim 1.

Claim 2: $z=\rho_{d} e^{i \theta}$, where $0<\theta<2 \pi$, is not a dominant singularity of $\mathbf{C}^{*}(z)$.

To prove Claim 2 we assume a contrario that $z=\rho_{d} e^{i \theta}, 0<\theta<2 \pi$ is a dominant singularity of $\mathbf{C}^{*}(z)$. Then the convergence radius of $\mathbf{C}^{*}(z)$ is $\rho_{d}$ and Theorem 3 implies that $\rho_{d}$ is also a dominant singularity of $\mathbf{C}^{*}(z)$, which contradicts Claim 1 , where we showed that $\rho_{d}$ is a removable singularity.

Therefore, in case of $\rho_{d}<\rho_{r}, \rho_{c^{*}} \neq \rho_{d}$. Consequently, Claim 1 and Claim 2 imply $\rho_{c^{*}}=\rho_{r}$.

We thus have the following two scenarios for $\rho_{s^{*}}$ :

$$
\rho_{s^{*}}= \begin{cases}\rho_{r} & \text { for } \quad \rho_{r}<\rho_{p} \\ \rho_{p} & \text { for } \quad \rho_{p} \leq \rho_{r}\end{cases}
$$

and obtain the three cases:

(I): $\rho_{c^{*}}=\rho_{r}$ and $\rho_{s^{*}}=\rho_{r} ;$

(II): $\rho_{c^{*}}=\rho_{r}$ and $\rho_{s^{*}}=\rho_{p}$ with $\rho_{p}<\rho_{r}$; 
(III): $\rho_{c^{*}}=\rho_{r}$ and $\rho_{s^{*}}=\rho_{p}$ with $\rho_{p}=\rho_{r}$

and Lemma 1 follows.

Lemma 2. The dominant singularity of $\mathbf{C}^{*}(z), \rho_{c^{*}}$, is unique.

Proof. Claim 0: $\mathbf{C}^{*}(z)$ is aperiodic.

It is clear that there always exists some irreducible secondary structure of length $n \geq$ 5. The coefficients of $\mathbf{C}^{*}(z)$ are weighted sums of these structures and the weights are, by construction, always strictly positive. Therefore, $\mathbf{C}^{*}(z)=\sum_{n \geq 0} \mathbf{c}^{*}(n) z^{n}$ has for $n \geq 5$ always strictly positive coefficients, i.e. $\mathbf{c}^{*}(n)>0$. Hence, there exist three indices $i<j<k$ such that $\mathbf{c}^{*}(i) \mathbf{c}^{*}(j) \mathbf{c}^{*}(k) \neq 0$ and $\operatorname{gcd}(j-i, k-i)=1$, therefore $\mathbf{C}^{*}(z)$ is aperiodic and Claim 0 is proved.

In view of eq. (3.1), we compute

$$
\begin{aligned}
& \mathbf{C}(z, v, p) \\
& =p v^{\alpha_{1}} z^{2}\left(z v^{\alpha_{2}}\right)^{3} \sum_{i \geq 0}\left(z v^{\alpha_{2}}\right)^{i}+\left(v^{\alpha_{3}}-v^{4 \alpha_{2}}\right) z^{4}+p z^{2} v^{\beta_{1}} \mathbf{C}(z, v, p)\left(\sum_{j \geq 0}\left(z v^{\beta_{2}}\right)^{j}\right)^{2} \\
& +p v^{\gamma_{1}}\left(z^{2} v^{\gamma_{2}}\right)\left(\mathbf{C}(z, v, p) v^{\gamma_{2}}\right)^{2}\left(\sum_{k \geq 0} z^{k}\right) \sum_{l \geq 0}\left(\mathbf{C}(z, v, p) v^{\gamma_{2}} \sum_{t \geq 0} z^{t}\right)^{l} .
\end{aligned}
$$

Setting $p=\frac{6}{16}, v=e^{\frac{1}{R T}}$ and $w=\mathbf{C}^{*}(z)=\sum_{n \geq 0} \mathbf{c}^{*}(n) z^{n}$ we obtain a power series equation

$$
w=G(z, w),
$$


where $G(z, w)=\sum_{m, n>0} g_{m, n} z^{m} w^{n}$ and $g_{m, n} \geq 0$. Indeed, since $\alpha_{3}>0$ and $\alpha_{2}<0$, we have $v^{4 \alpha_{2}}<v^{\alpha_{3}}$ and $v^{\alpha_{3}}-v^{4 \alpha_{2}}>0$. The other coefficients in eq. (2) are all positive, i.e. $v^{\mathcal{P}}>0, \mathcal{P}=\left\{\alpha_{1}, \alpha_{2}, \alpha_{3}, \beta_{1}, \beta_{2}, \gamma_{1}, \gamma_{2}\right\}$, implying $g_{m, n} \geq 0$, in particular, $g_{0,1}=0$.

Furthermore, $G(z, w)$ is bivariate power series which is absolutely convergent in a domain $\mathscr{D}$, such that $|z|<R_{1},|w|<R_{2}$. According to Theorem 9.4.4 of [5], there exist a unique function analytic in a neighborhood $|z|<\rho$ of $z=0$, where $\rho \leq R_{1}$, such that

$$
\mathbf{C}^{*}(0)=0 \quad \text { and } \quad G\left(z, \mathbf{C}^{*}(z)\right)-\mathbf{C}^{*}(z)=0, \quad \text { for } \quad|z|<\rho, \quad \mathbf{C}^{*}(z)<R_{2} .
$$

Furthermore Theorem 9.4.6 of [5] shows that the radius of convergence, $\rho=\rho_{c^{*}}$, of the solution of eq. (3.8), $\mathbf{C}^{*}(z)$, and the value $w_{\rho}=\lim _{z \rightarrow \rho} \mathbf{C}^{*}(z)$ satisfy the equations

$$
w_{\rho}=G\left(\rho, w_{\rho}\right) \quad \text { and } \quad 1=G_{w_{\rho}}\left(\rho, w_{\rho}\right) .
$$

According to Lemma 1, we have $\rho_{c^{*}}=\rho_{r}$ and thus

$$
w_{\rho}=\lim _{z \rightarrow \rho} \mathbf{C}^{*}(z)=\mathbf{C}^{*}\left(\rho_{r}\right)=\frac{-w_{1}^{*}\left(\rho_{r}\right)}{w_{2}^{*}\left(\rho_{r}\right)} \neq 0,+\infty
$$

We next show that $\mathbf{C}^{*}(z)$ has no other dominant singularities than $\rho_{r}$. 
To this end we note that $\mathbf{C}^{*}(z)$ converges at point $z=\rho_{r}$. Since $\mathbf{c}^{*}(n) \geq 0$, applying the triangular inequality, we have $\mathbf{C}^{*}\left(\rho_{r} e^{i \theta}\right) \leq \mathbf{C}^{*}\left(\rho_{r}\right)$ Therefore $\mathbf{C}^{*}(z)$ converges on the whole circle $|z|=\rho_{r}$. Since $\mathbf{C}^{*}(z)$ is aperiodic and $\mathbf{C}^{*}(z)=\sum_{n \geq 0} \mathbf{c}^{*}(n) z^{n}$ is convergent power series for any $z$ with $|z|=\rho_{r}$, the Daffodil Lemma of [3] implies

$$
\left|\mathbf{C}^{*}\left(\rho_{r} e^{i \theta}\right)\right|<\mathbf{C}^{*}\left(\rho_{r}\right)
$$

Taking the derivative in eq. (3.8), we derive

$$
\frac{d}{d z} \mathbf{C}^{*}(z)=\frac{d}{d \mathbf{C}^{*}} G\left(z, \mathbf{C}^{*}(z)\right) \frac{d}{d z} \mathbf{C}^{*}(z)+\frac{d}{d z} G\left(z, \mathbf{C}^{*}(z)\right)
$$

Thus we have

$$
w_{z}=\frac{G_{z}(z, w)}{1-G_{w}(z, w)}
$$

which implies that $\mathbf{C}^{*}(z)$ is indeed analytic as long as $G_{w}(z, w) \neq 1$.

Since $G(z, w)$ has non-negative coefficients, it is monotonously increasing and so is $G_{w}(z, w)$. Therefore, for points $\rho_{r} e^{i \theta}$, where $0<\theta<2 \pi$ we have

$$
\left|G_{w}\left(\rho_{r} e^{i \theta}, \mathbf{C}^{*}\left(\rho_{r} e^{i \theta}\right)\right)\right| \leq\left|G_{w}\left(\left|\rho_{r} e^{i \theta}\right|,\left|\mathbf{C}^{*}\left(\rho_{r} e^{i \theta}\right)\right|\right)\right|<\left|G_{w}\left(\rho_{r}, \mathbf{C}^{*}\left(\rho_{r}\right)\right)\right|=1,
$$

which implies that $\mathbf{C}^{*}(z)$ is analytic on the whole circle $|z|=\rho_{r}$ except of $\rho_{r}$. This proves that $\rho_{c^{*}}=\rho_{r}$ is unique. 
Lemma 3. The dominant singularity of $\mathbf{S}^{*}(z)$ is unique and there are the following three cases:

(I): If $\rho_{s^{*}}=\rho_{c^{*}}=\rho_{r}$, then $\rho_{r}$ is the unique dominant singularity of $\mathbf{S}^{*}(z)$;

(II): If $\rho_{s^{*}}=\rho_{p}<\rho_{r}$, then $\rho_{p}$ is the unique dominant singularity of $\mathbf{S}^{*}(z)$;

(III): If $\rho_{s^{*}}=\rho_{p}=\rho_{r}$, then $\rho_{p}$ is the unique dominant singularity of $\mathbf{S}^{*}(z)$;

Furthermore, in case of (II) and (III), the degree of the root, $\rho_{p}$ of the equation $1-\left(z+\mathbf{C}^{*}(z)\right)=0$ is exactly 1 .

Proof. According to Lemma 1, we need to distinguish the cases (I), (II) and (III).

(I), here $\rho_{r}$ is the unique dominant singularity of $\mathbf{C}^{*}(z)$. Then in view of eq. (3), the dominant singularity of $\mathbf{S}^{*}(z)$ comes from the circle $|z|=\rho_{r}$, whence $\rho_{r}$ is also the unique dominant singularity of $\mathbf{S}^{*}(z)$.

(II) in this case we have $\rho_{s^{*}}=\rho_{p}<\rho_{c^{*}}$ and the dominant singularities of $\mathbf{S}^{*}(z)$ all come from the circle $|z|=\rho_{p}$. We have

$$
\mathbf{S}^{*}(z)^{-1}=1-\left(z+\mathbf{C}^{*}(z)\right)
$$

and substituting $z=\rho_{p}$, we derive

$$
\left(\rho_{p}+\mathbf{C}^{*}\left(\rho_{p}\right)\right)=1
$$


Then $\mathbf{D}^{*}(z)=z+\mathbf{C}^{*}(z)=\sum_{n \geq 0} \mathbf{d}^{*}(n) z^{n}$ is an aperiodic power series with nonnegative coefficients. According to Lemma 1, we have

$$
\mathbf{D}^{*}\left(\rho_{p}\right)=\rho_{p}+\mathbf{C}^{*}\left(\rho_{p}\right)=1
$$

Since $\mathbf{d}^{*}(n) \geq 0$, applying the triangular inequality implies $\mathbf{D}^{*}\left(\rho_{p} e^{i \theta}\right) \leq \mathbf{D}^{*}\left(\rho_{p}\right)$ and $\mathbf{D}^{*}(z)$ converges on the whole circle $|z|=\rho_{p}$. Since $\mathbf{D}^{*}(z)$ is aperiodic the Daffodil Lemma of [3], guarantees

$$
\left|\mathbf{D}^{*}\left(\rho_{p} e^{i \theta}\right)\right|<\mathbf{D}^{*}\left(\rho_{p}\right)<1 \text {. }
$$

Therefore, $\left|\mathbf{D}^{*}\left(\rho_{p} e^{i \theta}\right)\right| \neq 1$, for $0<\theta<2 \pi$, whence the points on the circle $|z|=\rho_{p}$ other than the point $\rho_{p}$, are not singularities of $\mathbf{S}^{*}(z)$.

(III) here the dominant singularities of $\mathbf{S}^{*}(z)$ all come from the circle $|z|=\rho_{p}=\rho_{r}$. According (I) and (II), $\rho_{p}=\rho_{r}$ is an unique dominant singularity of $\mathbf{S}^{*}(z)$.

In case of (II) and (III) and in view of eq. (3), the denominator of $\mathbf{S}^{*}(z)$ is

$$
1-\left(z+\mathbf{C}^{*}(z)\right)
$$

and $1-\left(\rho_{p}+\mathbf{C}^{*}\left(\rho_{p}\right)\right)=0$. Taking the derivative of eq. (3.12), we obtain $(1+$ $\left.\frac{d}{d z} \mathbf{C}^{*}(z)\right)=0$. Since $\mathbf{C}^{*}(z)=\sum_{n \geq 0} \mathbf{c}^{*}(n) z^{n}$, where $\mathbf{c}^{*}(n)>0$, for $n \geq 5$, the derivative $\frac{d}{d z} \mathbf{C}^{*}(z)=\sum_{n \geq 1} n \mathbf{c}^{*}(n) z^{n-1}$, has also nonnegative coefficients. As a 
result we have $\frac{d}{d z} \mathbf{C}^{*}\left(\rho_{p}\right)>0$ and consequently $\left(1+\frac{d}{d z} \mathbf{C}^{*}\left(\rho_{p}\right)\right)>0$. We conclude that $\rho_{p}$ is not a multiple root of eq. (3.12), which completes the proof of Lemma 3 .

\section{The MAIn Result}

We consider the general composition scheme

$$
\mathcal{F}=\mathcal{G} \circ(u \mathcal{H}) \Longrightarrow \mathbf{F}(z, u)=g(u h(z))
$$

Assume that $g$ and $h$ have non-negative coefficients and that $h(0)=0$, so that the composition $g(h(z))$ is well-defined. We let $\rho_{g}$ and $\rho_{h}$ denote the radii of convergence of $g$ and $h$, and define

$$
\tau_{g}=\lim _{x \rightarrow \rho_{g}^{-}} g(x) \quad \text { and } \quad \tau_{h}=\lim _{x \rightarrow \rho_{h}^{-}} h(x) .
$$

Definition 1. 3] The composition scheme $\mathbf{F}(z, t)=g(t h(z))$ is said to be subcritical if $\tau_{h}<\rho_{g}$, critical if $\tau_{h}=\rho_{g}$, and supercritical if $\tau_{h}>\rho_{g}$.

We observe that

$$
\mathbf{S}^{*}(z, t)=\frac{1}{1-\left(z+t \mathbf{C}^{*}(z)\right)}=f(z) g(t h(z))
$$

where $f(z)=\frac{1}{1-z}, g(w)=\frac{1}{1-w}$ and $h(z)=\frac{\mathbf{C}^{*}(z)}{1-z}$. Furthermore, we set

$$
\mathbb{P}\left(X_{n}=t\right)=\mathbf{s}^{*}(n, t) / \mathbf{s}^{*}(n) .
$$


Since $0<\rho_{s^{*}} \leq \rho_{c^{*}}<1$, we restrict ourselves to the cases $0<\rho_{d}, \rho_{r}, \rho_{p}<1$.

Theorem 4. The distribution of irreducible substructures within energy-filtered $R N A$ secondary structures has the following distinct regimes:

(a) the subcritical regime: Both dominant singularities $z=\rho_{c^{*}}$ of $\mathbf{C}^{*}(z)$ and $z=\rho_{s^{*}}$ of $\mathbf{S}^{*}(z)$ are all exclusively a branch point (square root) singularity. Then $\mathbb{P}\left(X_{n}=t\right)$ satisfies a discrete limit law and

$$
\lim _{n \rightarrow \infty} \frac{\mathbf{c}^{*}(n)}{\mathbf{s}^{*}(n)}=\chi>0 .
$$

(b) the supercritical regime: The dominant singularity $z=\rho_{c^{*}}$ of $\mathbf{C}^{*}(z)$ is exclusively branch point singularity; the dominant singularity $z=\rho_{s^{*}}$ of $\mathbf{S}^{*}(z)$ is exclusively a pole; . Then the probability distribution of $\mathbb{P}\left(X_{n}=t\right)$, after standardization, satisfies a limiting Gaussian distribution and

$$
\lim _{n \rightarrow \infty} \frac{\mathbf{c}^{*}(n)}{\mathbf{s}^{*}(n)}=0
$$

(c) the critical regime: The dominant singularity $z=\rho_{c^{*}}$ of $\mathbf{C}^{*}(z)$ is exclusively branch point singularity, the dominant singularity $z=\rho_{s^{*}}$ of $\mathbf{S}^{*}(z)$ is simultaneously a branch point singularity and a pole. Then $\mathbb{P}\left(X_{n}=t\right)$ satisfies a local limit law whose density is a Rayleigh distribution and

$$
\lim _{n \rightarrow \infty} \frac{\mathbf{c}^{*}(n)}{\mathbf{s}^{*}(n)} \sim \frac{\chi^{\prime}}{n}
$$

where $\chi^{\prime}>0$. 
Proof. To prove the theorem we shall show how the three scenarios identified in Lemma 3 give rise to the three regimes.

(a): Let us begin with case (I) of Lemma 3, where we have $\rho_{c^{*}}=\rho_{s^{*}}=\rho_{r}$ and $\rho_{r}<\rho_{p}$ and both dominant singularities $z=\rho_{c^{*}}$ of $\mathbf{C}^{*}(z)$ and $z=\rho_{s^{*}}$ of $\mathbf{S}^{*}(z)$ are all exclusively a branch point (square root) singularity.

We set

$$
\begin{aligned}
& t_{0}(z)=\left(w_{1}^{*}(z)^{2}-4 w_{2}^{*}(z) w_{0}^{*}(z)\right) /\left(z-\rho_{r}\right), \\
& t_{1}(z)=\left(-w_{1}^{*}(z)\right) /\left(2 w_{2}^{*}(z)\right), \\
& t_{2}(z)=\left(\sqrt{t_{0}(z)}\right) /\left(2 w_{2}^{*}(z)\right)
\end{aligned}
$$

and express $\mathbf{C}^{*}(z)$ as

$$
\mathbf{C}^{*}(z)=t_{1}(z)+t_{2}(z)\left(z-\rho_{r}\right)^{\frac{1}{2}}
$$

The singular expansion of $\mathbf{C}^{*}(z)$ is obtained from the regular expansion of $t_{1}(z)$ and the singular expansion of $\left(z-\rho_{r}\right)^{\frac{1}{2}} t_{2}(z)$. Consequently,

$$
\mathbf{C}^{*}(z)=t_{1}\left(\rho_{r}\right)+t_{2}\left(\rho_{r}\right)\left(z-\rho_{r}\right)^{\frac{1}{2}}+O\left(z-\rho_{r}\right) .
$$

In view of $O\left(z-\rho_{r}\right)=o\left(\left(z-\rho_{r}\right)^{\frac{1}{2}}\right)$, Theorem VI.3 3] implies

$$
\left[z^{n}\right] \mathbf{C}^{*}(z) \sim t_{2}\left(\rho_{r}\right)\left[z^{n}\right]\left(z-\rho_{r}\right)^{\frac{1}{2}}
$$


Using Theorem VI.1 of [3], we obtain

$$
\left[z^{n}\right] \mathbf{C}^{*}(z) \sim k_{1} \cdot n^{-\frac{3}{2}} \cdot\left(\rho_{r}\right)^{-n} \cdot\left(1+O\left(\frac{1}{n}\right)\right), \quad \text { for some constant } k_{1} .
$$

Analogously we compute the singular expansion of $\mathbf{S}^{*}(z)$ as:

$$
\mathbf{S}^{*}(z)=d_{1}+d_{2}\left(z-\rho_{r}\right)^{\frac{1}{2}}+O\left(z-\rho_{r}\right), \quad \text { for some constants } d_{1}, d_{2} .
$$

Employing Theorem VI.1 of [3],

$$
\left[z^{n}\right] \mathbf{S}^{*}(z) \sim k_{2} \cdot n^{-\frac{3}{2}} \cdot\left(\rho_{r}\right)^{-n}\left(1+O\left(\frac{1}{n}\right)\right), \quad \text { for some constant } k_{2} .
$$

Therefore,

$$
\lim _{n \rightarrow \infty} \frac{\mathbf{c}^{*}(n)}{\mathbf{s}^{*}(n)} \sim \frac{k_{1} \cdot n^{-\frac{3}{2}} \cdot\left(\rho_{r}\right)^{-n}}{k_{2} \cdot n^{-\frac{3}{2}} \cdot\left(\rho_{r}\right)^{-n}}=\chi>0
$$

We now have $\rho_{r}<\rho_{p}$, furthermore, we have (eq. (4.3)) with $\rho_{g}=1$ and

$$
\tau_{h}=\lim _{z \rightarrow \rho_{h}^{-}} \frac{\mathbf{C}^{*}(z)}{1-z} .
$$

Since $0<\rho_{p}<1$ is a root of $1-\left(z+\mathbf{C}^{*}(z)\right)=0$, we observe $\rho_{p}+\mathbf{C}^{*}\left(\rho_{p}\right)=1$. $\mathbf{C}^{*}(z)$ is a power series with positive coefficients and thus as a function over [0,1[, continuous and monotone. As a result $\rho_{r}+\mathbf{C}^{*}\left(\rho_{r}\right)<1$ and $\tau_{h}=h\left(\rho_{r}\right)=\frac{\mathbf{C}^{*}\left(\rho_{r}\right)}{1-\rho_{r}}<1=\rho_{g}$, i.e. $\mathbf{S}^{*}(z, t)$ is governed by the subcritical paradigm. 
According to Proposition IX.1 [3], the quotient of the coefficients $\mathbf{s}^{*}(n, k)$ and $\mathbf{s}^{*}(n)$ satisfies

$$
\lim _{n \rightarrow \infty} \frac{\mathbf{s}^{*}(n, k)}{\mathbf{s}^{*}(n)}=q_{k}, \text { where } q_{k}=\frac{k g_{k} \tau_{h}^{k-1}}{g^{\prime}\left(\tau_{h}\right)} .
$$

Therefore the probability generating function of the limit distribution $\left(q_{k}\right)$ is given by

$$
q(t)=\frac{\operatorname{tg}^{\prime}\left(\tau_{h} t\right)}{g^{\prime}\left(\tau_{h}\right)}
$$

and $\mathbb{P}\left(X_{n}=t\right)=\frac{\mathbf{s}^{*}(n, k)}{\mathbf{s}^{*}(n)}$ satisfies a discrete limit law as asserted.

Ad (b): We have the case (II) of Lemma 3, we have $\rho_{c^{*}}=\rho_{r}, \rho_{s^{*}}=\rho_{p}$ and $\rho_{p}<\rho_{r}$, the dominant singularity $z=\rho_{c^{*}}$ of $\mathbf{C}^{*}(z)$ is exclusively branch point singularity; the dominant singularity $z=\rho_{s^{*}}$ of $\mathbf{S}^{*}(z)$ is exclusively a pole with the relation.

In analogy to our arguments in (a) we derive

$$
\begin{aligned}
& \mathbf{s}^{*}(n) \sim k_{3} \cdot 1 \cdot\left(\rho_{s^{*}}\right)^{-n} \cdot\left(1+O\left(\frac{1}{n}\right)\right), \quad \omega_{1} \geq 1 \text { and } \omega_{1} \in \mathbb{N}, \\
& \mathbf{c}^{*}(n) \sim k_{1} n^{-\frac{3}{2}} \cdot\left(\rho_{c^{*}}\right)^{-n} \cdot\left(1+O\left(\frac{1}{n}\right)\right),
\end{aligned}
$$

for constants $k_{3}, k_{1}$ and clearly,

$$
\frac{\mathbf{c}^{*}(n)}{\mathbf{s}^{*}(n)} \sim \frac{k_{1} \cdot n^{-\frac{3}{2}}\left(\rho_{c^{*}}\right)^{-n}}{k_{3} \cdot 1 \cdot\left(\rho_{s^{*}}\right)^{-n}}=\frac{k_{1}}{k_{3}} \cdot n^{-\frac{3}{2}}\left(\frac{\rho_{s^{*}}}{\rho_{c^{*}}}\right)^{n} \sim 0 .
$$


We now have $\rho_{c^{*}}>\rho_{p}=\rho_{s^{*}}$ and since for $0<\rho_{p}<1$ as well as $\rho_{p}+\mathbf{C}^{*}\left(\rho_{p}\right)=1$, analogous monotonicity arguments imply $\rho_{c^{*}}+\mathbf{C}^{*}\left(\rho_{c^{*}}\right)>1$. As a result

$$
\tau_{h}=h\left(\rho_{c^{*}}\right)=\frac{\mathbf{C}^{*}\left(\rho_{c^{*}}\right)}{1-\rho_{c^{*}}}>1=\rho_{g}
$$

i.e. $\mathbf{S}^{*}(z, t)$ is governed by the supercritical paradigm. According to Proposition IX.6 [3], $\mathbb{P}\left(X_{n}=k\right)=\mathbf{s}^{*}(n, k) / \mathbf{s}^{*}(n)$ satisfies a Gaussian limit law with speed of convergence $O(1 / \sqrt{n})$.

Ad (c): We consider finally case (III) of Lemma 3, where we have $\rho_{c^{*}}=\rho_{r}$ and $\rho_{s^{*}}=\rho_{p}$ with $\rho_{p}=\rho_{r}$, the dominant singularity $z=\rho_{c^{*}}$ of $\mathbf{C}^{*}(z)$ is exclusively branch point singularity, the dominant singularity $z=\rho_{s^{*}}$ of $\mathbf{S}^{*}(z)$ is simultaneously a branch point singularity and a pole.

Then the singular expansion of $\mathbf{S}^{*}(z)$ is given by:

$$
\mathbf{S}^{*}(z)=d_{3}+d_{4}\left(z-\rho_{s^{*}}\right)^{-\frac{1}{2}}+O\left(z-\rho_{s^{*}}\right)
$$

for constant $d_{3}$ and $d_{4}$. We compute

$$
\mathbf{s}^{*}(n) \sim k_{4} \cdot n^{-\frac{1}{2}} \cdot\left(\rho_{s^{*}}\right)^{-n} \cdot\left(1+O\left(\frac{1}{n}\right)\right)
$$

and for constant $k_{4}$. For $\mathbf{c}^{*}(n)$ we derive the asymptotic expression

$$
\mathbf{c}^{*}(n) \sim k_{1} \cdot n^{-\frac{3}{2}} \cdot\left(\rho_{c^{*}}\right)^{-n} \cdot\left(1+O\left(\frac{1}{n}\right)\right) .
$$


Consequently,

$$
\frac{\mathbf{c}^{*}(n)}{\mathbf{s}^{*}(n)} \sim \frac{k_{1} \cdot n^{-\frac{3}{2}} \cdot\left(\rho_{c^{*}}\right)^{-n}}{k_{4} \cdot n^{-\frac{1}{2}} \cdot\left(\rho_{s^{*}}\right)^{-n}}=\frac{\chi^{\prime}}{n} \sim 0, \chi^{\prime}=\frac{k_{1}}{k_{4}}
$$

In view of $\rho_{r}=\rho_{p}$ we obtain $\frac{\mathbf{C}^{*}\left(\rho_{r}\right)}{1-\rho_{r}}=1$ and

$$
\tau_{h}=h\left(\rho_{r}\right)=\frac{\mathbf{C}^{*}\left(\rho_{r}\right)}{1-\rho_{r}}=1=\rho_{g}
$$

whence $\mathbf{S}^{*}(z, t)$ belongs to the critical paradigm. The critical composition is $\mathbf{S}^{*}(z, t)=$ $f(z) g(t h(z))$, where $h(z)$ and $g(z)$ have singular exponent $\lambda=\frac{1}{2}$ and $\lambda^{\prime}=1$, where $\lambda<\lambda^{\prime}$. We proceed by applying Proposition IX.24 of [3], from which we can conclude that the normalized r.v. $X_{n} / \sqrt{n}$ satisfies a local limit law whose density is given by a Rayleigh law [2, 3]. To be specific we have

$$
\mathbb{P}\left(X_{n}=k\right)=\frac{\left[z^{k}\right] g(z)}{\left[z^{n}\right] g(h(z))}\left[z^{n}\right] h(z)^{k},
$$

and the singular expansion of $g(z), h(z)$ and $g(h(z))$ are respectively given by

$$
\begin{aligned}
g(z) & =(1-z)^{-1}, \\
h(z) & =\tau_{h}-h_{\frac{1}{2}}\left(1-\frac{z}{\rho_{r}}\right)^{\frac{1}{2}}+O\left(1-\frac{z}{\rho_{r}}\right), \\
g(h(z)) & =m_{0}-m_{-\frac{1}{2}}\left(1-\frac{z}{\rho_{r}}\right)^{-\frac{1}{2}}+O(1) .
\end{aligned}
$$


Thus we obtain

$$
\left[z^{k}\right] g(z) \sim(1)^{-k} \quad \text { and } \quad\left[z^{n}\right] g(h(z)) \sim m_{-\frac{1}{2}} \cdot \frac{n^{-\frac{1}{2}}}{\Gamma\left(\frac{1}{2}\right)} \cdot\left(\rho_{r}\right)^{-n}
$$

We next employ eq. (103) of Theorem IX.16 of [3] for $k=x n^{\frac{1}{2}}$, where $x$ is contained in any compact subinterval of $(0,+\infty)$. Then

$$
\left[z^{n}\right] h(z)^{k} \sim\left(\rho_{r}\right)^{-n} \cdot \frac{1}{n} \cdot \operatorname{Ray}\left(h_{\frac{1}{2}} x ; \frac{1}{2}\right),
$$

where $\operatorname{Ray}\left(x ; \frac{1}{2}\right)=\frac{x}{2} \exp \left(-\frac{x^{2}}{4}\right)$ is the Rayleigh density function. Hence

$$
\begin{aligned}
\mathbb{P}\left(X_{n}=k\right) & \sim \frac{\Gamma\left(\frac{1}{2}\right)}{m_{-\frac{1}{2}}} \frac{1}{\sqrt{n}} \operatorname{Ray}\left(h_{\frac{1}{2}} x ; \frac{1}{2}\right) \\
& =\frac{h_{\frac{1}{2}} \Gamma\left(\frac{1}{2}\right)}{2 m_{-\frac{1}{2}}} \cdot \frac{k}{n} \cdot \exp \left(-\frac{\left(h_{\frac{1}{2}}\right)^{2}}{4} \frac{k^{2}}{n}\right)
\end{aligned}
$$

and the proof of the theorem is complete.

\section{Discussion}

In this paper we demonstrated that the particular energy parametrization of RNA secondary structures affects the class of minimum free energy structures generated by DP-mfe folding algorithms in a subtle way. Minimal changes in parametrization can induce significant changes to the class of mfe-structures. We characterized the combinatorial impact of these changes in terms of the distribution of irreducible 
substructures which has practical implications on algorithmic level, i.e. for the effect of sparsification of mfe DP-folding of such structures.

We find the following dichotomy: the distribution of irreducible substructures either is a discrete limit law or a central limit law. In the former case mfe-structures contain only finitely many irreducible substructures and the ratio of irreducible mfestructures over all mfe-structures becomes in the limit of long sequences a positive constant. While this means "just" a constant time reduction for the sparsification of the DP-routine, the reduction is typically in the order of $90 \%$ [8] and consequently still of practical interest. In the latter case, the fact that the central limit distribution has a mean that scales linearly with sequence length alone implies the these mfestructures contain a large number of very small irreducible substructures. As these irreducibles are small it becomes more and more unlikely to realize a mfe structure as an irreducible. As a result sparsification has somewhat "maximal" effect, i.e. a linear reduction in time and space [19].

From the work of [4] we know that a natural RNA structure has a finite $5^{\prime}-3^{\prime}$ distance. This means that natural RNA structures contain only a finite number of irreducible substructures. In the context of our dichotomy result this means that sparsification of "realistically" parameterized mfe structures leads to a constant time reduction, in accordance with the findings in [8]. 
TABlE 1. Parameters of (a) (subcritical regime) and (b) (supercritical regime).

\begin{tabular}{|l|l|l|l|l|l|l|l|}
\hline & $\alpha_{1}$ & $\alpha_{2}$ & $\alpha_{3}$ & $\beta_{1}$ & $\beta_{2}$ & $\gamma_{1}$ & $\gamma_{2}$ \\
\hline subcritical & -5 & -0.01 & 7.53 & 4 & -1 & -3.4 & -0.6 \\
\hline supercritical & -5 & -0.01 & 7.53 & 2 & -1 & -10 & -3 \\
\hline
\end{tabular}

At the transition point, where the distribution shifts from a discrete to a central limit law, a local limit law exists. Its density function is that of a Rayleigh distribution. It is easy to "test" our main theorem for the subcritical and supercritical regimes, see Fig. 5, i.e. to sample the predicted limit laws. The particular parameters for the two scenarios displayed in Fig. 5 are given in Tab. 1, By construction it is practically impossible to localize the transition and sample the Rayleigh law.

In Fig. 6 we detail two typical RNA secondary structures sampled from the two regimes. Instead of presenting these structures as diagrams we map them into trees, where nodes represent irreducible substructures and edges are being drawn if irreducibles are nested. The tree representation shows clearly that a small variation of energy parameters can have a dramatic effect on the mfe structures. It is also evident why sparsification works much more efficient in the supercritical regime. Namely, in this case the irreducibles are less complex which implies that it becomes increasingly unlikely to find any candidates. 


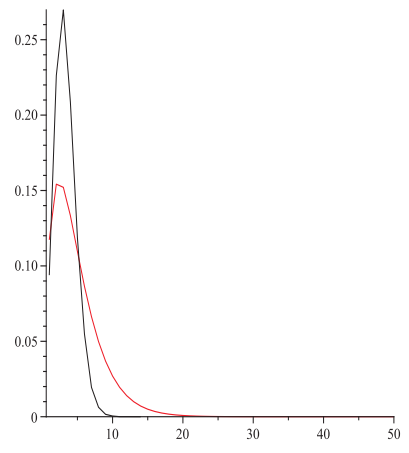

(a)

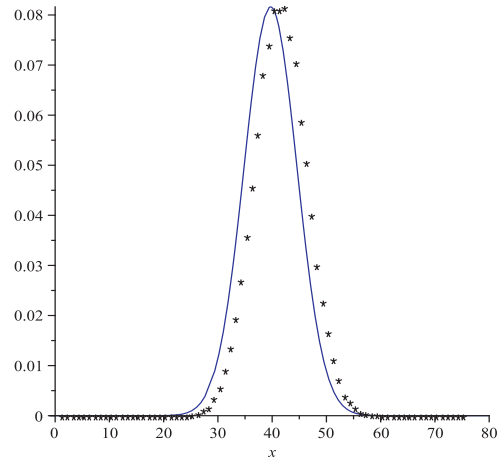

(b)

FIGURE 5. The subcritical regime $(a)$ : sampling $10^{5}$ structures of length $n=700$ (black) versus the discrete limit law (red) as predicted by Theorem 4. The supercritical regime $(b)$ : sampling $10^{6}$ structures of length $n=10^{3}$ (black stars) versus the central limit distribution as predicted by Theorem 4 (blue line).

\section{ACKnowledgments.}

We are grateful to Fenix W.D. Huang for his help with the sampling curves of Figure. 5.

\section{REFERENCES}

[1] R. Backofen, D. Tsur, S. Zakov, and M. Ziv-Ukelson, (2011), Sparse RNA folding: Time and space efficient algorithms, J. Disc. Algor., 9(1), 12-31.

[2] C. Banderier, P. Flajolet, G. Schaeffer, and M. Soria, (2001), Random maps, coalescing saddles, singularity analysis, and airy phenomena, Random Struct. Algor., 19, 194-246. . 


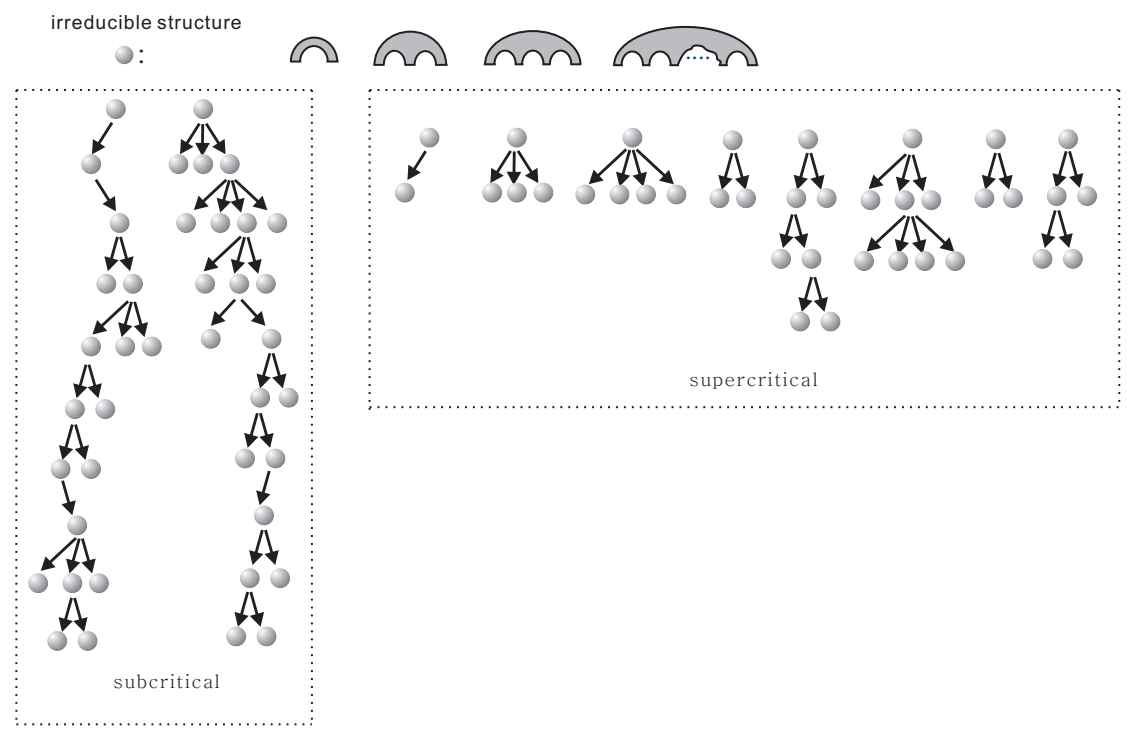

FIGURE 6. Tree representation of structures in the two regimes: a typical subcritical structure (left) and a typical supercritical structure (right). One node in a tree represents an irreducible substructure and an edge visualizes the nesting relation.

[3] P. Flajolet and R. Sedgewick, (2009), Analytic Combinatorics, Cambridge University Press New York.

[4] H.S.W. Han and C.M. Reidys, (2012), The 5'-3' distance of rna secondary structures, Jour. Comp. Biol., to appear.

[5] E. Hille, (1962), Analytic Function Theory, Volume I, Chelsea Publishing Company.

[6] I.L. Hofacker, W. Fontana, P.F. Stadler, L.S. Bonhoeffer, M. Tacker, and P. Schuster, (1994), Fast folding and comparison of RNA secondary structures, Monatsh. Chem., 125, 167-188.

[7] J.A. Howell, T.F. Smith, and M.S. Waterman, (1980), Computation of generating functions for biological molecules, SIAM J. Appl. Math., 39, 119-133.

[8] F.W.D. Huang and C.M. Reidys, (2012), On the combinatorics of sparsification, http://arxiv.org/abs/1201.0308v2

[9] D. Kleitman, (1970), Proportions of irreducible diagrams, Studies in Appl. Math., 49, 297-299. 
[10] D. Mathews, J. Sabina, M. Zuker, and D.H. Turner, (1999), Expanded sequence dependence of thermodynamic parameters improves prediction of RNA secondary structure, J. Mol. Biol., 288, 911-940.

[11] J.S. McCaskill, (1990), The equilibrium partition function and base pair binding probabilities for RNA secondary structure, Biopolymers, 29, 1105-1119.

[12] R. Nussinov, G. Piecznik, J.R. Griggs, and D.J. Kleitman, (1978), Algorithms for loop matching, SIAM J. Appl. Math., 35(1), 68-82.

[13] J. SantaLucia, (1996), Jr. biochemistry, 35, 3555.

[14] T.F. Smith and M.S. Waterman, (1978), RNA secondary structure, Math. Biol., 42, 31-49.

[15] E. C. Titchmarsh, (1939), The theory of functions.

[16] M.S. Waterman, (1978), Secondary structure of single-stranded nucleic acids, Adv. Math. (Suppl. Studies), 1, 167-212.

[17] M.S. Waterman, (1979), Combinatorics of RNA hairpins and cloverleafs, Stud. Appl. Math., 60, 91-96.

[18] M.S. Waterman and W.R. Schmitt, (1994), Linear trees and RNA secondary structure, Discr. Appl. Math., 51, 317-323.

[19] Y. Wexler, C. Zilberstein, and M. Ziv-Ukelson, (2007), A study of accessible motifs and RNA complexity, J. Comput. Biol., 14(6), 856-872.

[20] M. Zuker, (1989), On finding all suboptimal foldings of an RNA molecule, Science, 244, 48-52.

[21] M. Zuker and P. Stiegler, (1981), Optimal computer folding of larger RNA sequences using thermodynamics and auxiliary information, Nucleic Acids Res., 9, 133-148. 